\title{
BCG Vaccination and Immunity against COVID-19: A Fact or Fiction
}

\author{
Rajendra Prasad ${ }^{1}$ Rishabh Kacker ${ }^{1}$ Huda Shamim ${ }^{1}$ \\ 1Department of Pulmonary Medicine, Era's Lucknow Medical \\ College \& Hospital, Lucknow, Uttar Pradesh, India \\ ${ }^{2}$ Department of General Medicine, Dr. Ram Manohar Lohia Institute \\ of Medical Sciences, Lucknow, Uttar Pradesh, India
}

Ann Natl Acad Med Sci (India):2020;56:239-241
The first case of the novel Coronavirus disease, named COVID-19, was reported in Wuhan, China, on December 31, 2019. The illness has variable manifestations, ranging from common cold to the more severe Middle East respiratory syndrome (MERS) and severe acute respiratory syndrome (SARS). While the mortality rates of SARS and MERS was estimated to be approximately $12 \%$ and $30 \%$, respectively, the mortality rates in cases of COVID-19 is estimated to be much lower at around 4.03\%. ${ }^{1}$ Nevertheless, the pandemic of COVID-19 is an issue that needs to be taken seriously. However, there are striking differences on how COVID-19 is behaving in different countries. For instance, in Italy, where even though social interactions were severely curtailed, the mortality is quite high as compared with Japan, where the mortality is low despite not adopting restrictive social isolation measurements. Even in India, which is the second most populous country in the world, the mortality rates are pretty low as compared with Italy and other European nations. While these differences have been attributed to cultural norms as well as differences in medical care standards, an alternative explanation could be the national policy on Bacillus Calmette-Guerin (BCG) vaccination.

The BCG vaccine is a live attenuated strain derived from an isolate of Mycobacterium bovis. The vaccine has existed for more than 80 years and is one of the most widely used of all the current vaccines. Worldwide, more than 100 million children receive BCG each year. ${ }^{2}$ BCG vaccine was developed over a period of 13 years, from 1908, by Albert Calmette and Camille Guerin. The original BCG vaccine strain was formerly distributed by the Pasteur Institute of Paris and subcultured in different countries using different culture conditions that were not standardized. ${ }^{3}$ A limited rollout of vaccination was started in India in 1948 in an attempt to lower the tuberculosis (TB) burden in the country. By 1955-56, the mass campaign had covered all states of India. A single dose of BCG vaccine is required to provide lifetime immunity. Booster doses of BCG vaccine are not recommended. BCG is preferably given at birth to provide protection in the early years when infection can often lead to diseases such as miliary TB or tubercular meningitis. By the late 1940s, several studies had appeared, providing evidence in favor of the utility of BCG in protection against TB. In 1950s, major trials were set up by the Medical Research Council in the United Kingdom and by the Public Health Service in the United States. Soon it became evident that the procedure employed in the United Kingdom was highly efficacious against TB, ${ }^{4}$ whereas that in the United States provided little or no protection..$^{5}$ On the basis of these results, health agencies recommended BCG as a routine for tuberculin-negative adolescents in the United Kingdom, whereas BCG was not recommended for routine use in the United States. The majority of the world followed the lead of Europe and the WHO and introduced routine BCG vaccination according to various schedules, whereas the United States and the Netherlands decided against the use of BCG vaccination.

Due to the variability in results regarding the efficacy of BCG vaccination in the previous trials conducted, a large-scale, community-based double blind randomized controlled trial was performed in Chingleput district of South India to evaluate the protective effect of BCG against bacillary forms of pulmonary TB. The findings at 15 years showed that in this population with high-infection rates and high-nonspecific sensitivity, BCG did not offer any protection against adult forms of bacillary pulmonary TB. ${ }^{6}$ Despite the backlash caused by the above study, BCG vaccine is still a part of the national immunization program in India. A plethora of data from studies provide strong evidence for BCG's ability to protect against a wide range of infections other than $\mathrm{TB}$, including nontuberculous mycobacteria (NTM), leprosy and other bacteria (e.g., Shigella flexneri), viruses (e.g., vaccinia virus), protozoa (e.g., malaria), allergies, and eczema. Interestingly, the BCG's immunomodulatory properties are
Address for correspondence Rajendra Prasad, MD, DTCD, MBBS, A-28, Sector J, Aliganj, Lucknow 226024, Uttar Pradesh, India (e-mail: rprasadkgmc@gmail.com).
DOI https://doi.org/ $10.1055 / \mathrm{s}-0040-1718860$ ISSN 0379-038X. (c) 2020. National Academy of Medical Sciences (India).

This is an open access article published by Thieme under the terms of the Creative Commons Attribution-NonDerivative-NonCommercial-License, permitting copying and reproduction so long as the original work is given appropriate credit. Contents may not be used for commercial purposes, or adapted, remixed, transformed or built upon. (https://creativecommons.org/ licenses/by-nc-nd/4.0/).

Thieme Medical and Scientific Publishers Pvt. Ltd. A-12, 2nd Floor, Sector 2, Noida-201301 UP, India 
routinely exploited in the treatment of bladder cancer and melanoma. $^{7}$

The possible link between BCG vaccination and its protective effects against COVID-19 came into limelight when the global spread of COVID-19 was correlated with the data from the world BCG Atlas, and the study concluded that countries such as India, China and Japan with a policy of universal BCG vaccination have had a lower number of cases than those in Spain, France, and Switzerland which discontinued their universal vaccine policies. While countries such as the United States, Italy, and Netherlands have yet to adopt universal policies. This study also tries to explain the anomalous case of Iran, where the universal immunization program for BCG was only recently started in 1984 , unlike China and India, whose program goes back to the late 1940s. Hence, most deaths in Iran comprise elderly people who did not receive any immunization under the program. ${ }^{8}$ The possible explanation as to how BCG vaccination can provide immunity against the COVID-19 is that this vaccine has been shown to produce positive "heterologous" or nonspecific immune effects, leading to improved response against other nonmycobacterial pathogens. In a study conducted by Mathurin et al, the BCG-vaccinated mice infected with the vaccinia virus were protected by increased production of IFN-Ỳ by CD4+ cells. ${ }^{9}$ BCG vaccination has also been shown to provide broad protection against viral infections and sepsis, ${ }^{10}$ raising the possibility that the protective effect of the vaccine might not be directly related to actions on COVID-19 but on associated co-occurring infections or sepsis. In a recent study conducted by Miller et al, they found evidence that BCG vaccination seems to significantly reduce mortality associated with COVID-19. They also concluded that earlier a country established a BCG vaccination policy, the stronger the reduction in their number of deaths per million inhabitants, which is consistent with the idea that protecting the elderly population might be crucial in reducing mortality. ${ }^{11}$ Even though the data might suggest the positive role of BCG immunization against COVID-19, we must take into consideration that these are just mere observations with limited evidence, and hence not a proven entity.

Soon after the claim of BCG vaccination to confer immunity against COVID-19 was made, it was strongly opposed by other researchers, who questioned the methodology and the extent of COVID-19 spread globally at the time the study was conducted and some of the presumptions were put forward. They pointed out the fact that there is a danger in citing that a century-old vaccine may boost immunity in individuals and provide nonspecific protection against COVID-19 without any clear evidence. It was also brought to attention that at the time when analysis was done, India had a fewer number of cases as compared with the present scenario; hence, such speculations should not be made unless and until such observations are proven by trials. Another major drawback of the study was that the countries that were selected were arbitrary. Even Australia and Germany have a much lower morbidity and mortality rate and have no universal BCG immunization policy; hence, BCG solely cannot be the answer.
Also, health systems' capacities, infrastructure, staffing, ICUs, ventilators, and innate immunity of people are all confounding factors which also need to be taken into account. ${ }^{12}$ And although inconclusive, the prevailing weather and temperature also play a part in the spread and containment of an epidemic, which is an idea that needs to be researched too, since most tropical countries have had low caseloads. Whether BCG will be effective remains unknown, as it is based on the findings from the ecological studies which are supposed to be a form of weak evidence. ${ }^{13}$ To settle this dispute, controlled trials are required. Currently, a randomized control trial called BRACE trial is undergoing in Australia in which 4170 health care workers are being enrolled to evaluate the efficacy of BCG vaccination in providing immunity against COVID-19. ${ }^{13}$ Similarly, a trial in Netherlands aims to evaluate the efficacy of BCG vaccination in reducing health care workers' absenteeism in SARS-CoV-2. ${ }^{14}$ An upcoming trial by the Serum Institute of India is currently under consideration to test the efficacy of BCG in high-risk COVID-19 groups in Maharashtra, India. ${ }^{15}$ The results of these trials will be able to prove or disprove about the protection of BCG vaccination against COVID-19.

\section{Authors' Contributions}

R.P.: Chief author and editor; R.K.: Compilation of data; H.S.: Data collection and editing; N.G.: Data collection and tabulation.

\section{Conflict of Interest}

None declared.

\section{References}

1 Baud D, Qi X, Nielsen-Saines K, Musso D, Pomar L, Favre G. Real estimates of mortality following COVID-19 infection. Lancet Infect Dis 2020;20(7):773

2 TrunzBB, FineP,DyeC.Effect ofBCG vaccination on childhood tuberculous meningitis and miliary tuberculosis worldwide: a metaanalysis and assessment of cost-effectiveness. Lancet 2006; 367(9517):1173-1180

3 Recommendations to assure the quality, safety and efficacy of BCG vaccines. WHO Technical Report Series No. 979, 2013. Available at: https://www.who.int/biologicals/areas/vaccines/ TRS_979_Annex_3.pdf. Accessed July 20, 2020

4 Hart PD, Sutherland I. BCG and vole bacillus vaccines in the prevention of tuberculosis in adolescence and early adult life. BMJ 1977;2(6082):293-295

5 Comstock GW, Palmer CE. Long-term results of BCG vaccination in the southern United States. Am Rev Respir Dis 1966; 93(2):171-183

6 Tuberculosis Research Centre. Fifteen year follow up of trial of BCG vaccines in south India for tuberculosis prevention. Tuberculosis Research Centre (ICMR), Chennai. Indian J Med Res 1999;110:56-69

7 Report on BCG vaccine use for protection against mycobacterial infections including tuberculosis, leprosy, and other nontuberculous mycobacteria (NTM) infections. The SAGE Working Group on BCG Vaccines and WHO Secretariat. September 22, 2017. Available at: https://www.who.int/immunization/sage/ meetings/2017/october/1_BCG_report_revised_version_ online.pdf. Accessed June 05, 2020

8 New York Institute of technology. TB Vaccine Could Be a Valuable Weapon in COVID-19 Fight. Available at: https://www. 
forbes.com/sites/madhukarpai/2020/04/12/bcg-againstcoronavirus-less-hype-and-more-evidence-please/. Accessed May 05, 2020

9 Mathurin KS, Martens GW, Kornfeld H, Welsh RM. CD4 T-cell-mediated heterologous immunity between mycobacteria and poxviruses. J Virol 2009;83(8):3528-3539

10 Moorlag SJCFM, Arts RJW, van Crevel R, Netea MG. Non-specific effects of BCG vaccine on viral infections. Clin Microbiol Infect 2019;25(12):1473-1478

11 Miller A, Reandelar MJ, Fasciglione K, et al. Correlation between universal BCG vaccination policy and reduced morbidity and mortality for COVID-19: an epidemiological study. MedRxiv 2020 Doi: $10.1101 / 2020.03 .24 .20042937$

12 Forbes. Available at: https://www.nyit.edu/box/features/tb_ vaccine_could_be_a_valuable_weapon_in_covid_19_fight. Accessed May 06, 2020
13 Faust L, Huddart S, MacLean E, Svadzian A. Universal BCG Vaccination and Protection Against COVID-19: Critique of an Ecological Study. Available at: https://naturemicro biologycommunity.nature.com/users/36050-emily-maclean/posts/ 64892-universal-bcgvaccination-and-protection-against-covid-1 9-critique-of-an-ecological-study. Accessed April 15, 2020

14 ClinicalTrials.gov. BCG Vaccination to Protect Healthcare Workers Against COVID-19 (BRACE). Available at: https://clinicaltrials.gov/ct2/show/NCT04327206. Accessed April 27, 2020

15 ClinicalTrials.gov. Reducing Health Care Workers Absenteeism in Covid-19 Pandemic Through BCG Vaccine (BCG-CORONA). Available at: https://clinicaltrials.gov/ct2/show/NCT04328441. Accessed May 05, 2020 